\title{
Experimental demonstration of phase measurement precision beating standard quantum limit by projection measurement
}

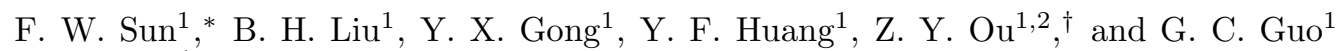 \\ ${ }^{1}$ Key Laboratory of Quantum Information, University of Science and \\ Technology of China (CAS), Hefei 230026, People's Republic of China. \\ ${ }^{2}$ Department of Physics, Indiana University-Purdue University Indianapolis \\ 402 N. Blackford Street, Indianapolis, IN 46202
}

(Dated: October 30, 2018)

\begin{abstract}
We propose and demonstrate experimentally a projection scheme to measure the quantum phase with a precision beating the standard quantum limit. The initial input state is a twin Fock state $|N, N\rangle$ proposed by Holland and Burnett [Phys. Rev. Lett. 71, 1355 (1993)] but the phase information is extracted by a quantum state projection measurement. The phase precision is about $1.4 / N$ for large photon number $N$, which approaches the Heisenberg limit of $1 / N$. Experimentally, we employ a four-photon state from type-II parametric down-conversion and achieve a phase uncertainty of $0.291 \pm 0.001$ beating the standard quantum limit of $1 / \sqrt{N}=1 / 2$ for four photons.

PACS numbers: 42.50.Dv, 03.65.Ta, 07.60.Ly.
\end{abstract}

The measurement of the magnitude of a physical quantity is one of the main tasks of modern physics. The key question is what precision can be achieved in the measurement. Principally, this is governed by the laws of quantum mechanics. For example, the precision of the quantum phase measurement can be intuitively understood from the Heisenberg uncertainty principle for the phase and photon number [1] as

$$
\Delta \phi \Delta N \geq 1
$$

where $\Delta \phi$ and $\Delta N$ are the fluctuation for the phase and photon number. Therefore, the shot noise of $\Delta N=$ $\sqrt{\langle N\rangle}$ from a laser in coherent state gives rise to the shot-noise limit or the so-called standard quantum limit [2] for the phase measurement as $\Delta \phi \gtrsim 1 / \sqrt{\langle N\rangle}$ with an average photon number of $\langle N\rangle$.

However, the standard quantum limit is not the ultimate limit for the precision in phase measurement. It has been surpassed with squeezed state based interferometry [3, 4]. The ultimate limit was proven [5, 6] to be the Heisenberg limit 7| of $1 /\langle N\rangle$. A number of schemes were proposed [3, 6, 8, 9, 10, 11] that can reach this limit. But none of them was realized experimentally, primarily due to the fragile effect of loss in the system.

Recently, research focus is shifted to the maximally entangled photon-number state (MES) or the so-called NOON state [6, 10, 12, 13, 14] in the form $(|N, 0\rangle+$ $|0, N\rangle) / \sqrt{2}$, which, due to a multi-photon interference effect, leads to a multi-photon detection probability of

$$
P_{M E S}(\phi)=(1+\cos N \phi) / 2 .
$$

The advantage of using MES is that it is not as sensitive to loss as the squeezed states. Loss will simply reduce the success probability and increase the number of photons required for phase measurement, but in a linear fashion. It has been demonstrated that the above super-resolved phase dependence can be achieved without the need of a NOON state. However, as recently pointed out 15,
16], the super-resolved phase dependence in Eq.(2) does not necessarily lead to a phase measurement precision exceeding the standard quantum limit.

In this letter, we will study the problem of precision phase measurement with a different approach. We will combine the concept of twin-photon state [8, 11] with recently developed quantum state projection method [16]. We find that the new measurement scheme allows us to achieve a phase measurement precision that is close to the Heisenberg limit. We demonstrate the feasibility of the scheme with two-photon state and four-photon state and obtain a phase measurement precision of $0.501 \pm 0.001$ and $0.291 \pm 0.001$ for these states, respectively. These values are significantly better than $1 / \sqrt{2}$ and $1 / 2$ set by the standard quantum limit.

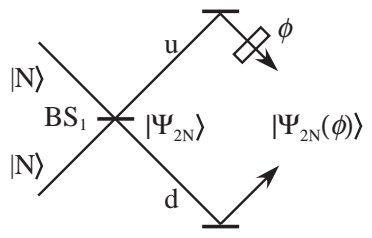

(a)

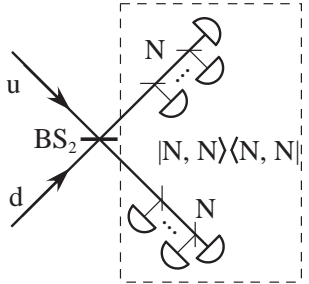

(b)
FIG. 1: Projection measurement for phase measurement with a twin-Fock state. (a) Generation of the state $\left|\Psi_{2 N}(\phi)\right\rangle$ from a twin-Fock state; (b) Projection measurement of $\mathbb{P}=$ $\left|\Psi_{2 N}\right\rangle\left\langle\Psi_{2 N}\right|$. The dashed box is a projection to $|N, N\rangle$.

Theory - Consider a two-mode state that is generated by injecting a twin Fock state $|N, N\rangle$ into a 50:50 beam splitter [8] as shown in Fig.1. The two-mode state has the form of:

$$
\begin{gathered}
\left|\Psi_{2 N}\right\rangle=\sum_{k=0}^{N}(-1)^{N-k}\left[\left(\begin{array}{c}
2 k \\
k
\end{array}\right)\left(\begin{array}{c}
2 N-2 k \\
N-k
\end{array}\right)\left(\frac{1}{2}\right)^{2 N}\right]^{1 / 2} \\
\times|2 k\rangle_{u}|2 N-2 k\rangle_{d}
\end{gathered}
$$


where $|2 k\rangle_{u}|2 N-2 k\rangle_{d}$ denote $2 k$ photons and $2 N-2 k$ photons in the up and down modes, respectively. This state has been studied extensively in Refs. [8, 11]. The photon number variance is

$$
\begin{aligned}
\left\langle\Delta^{2} N\right\rangle_{u} & =\left\langle\hat{a}_{u}^{\dagger} \hat{a}_{u} \hat{a}_{u}^{\dagger} \hat{a}_{u}\right\rangle-\left\langle\hat{a}_{u}^{\dagger} \hat{a}_{u}\right\rangle\left\langle\hat{a}_{u}^{\dagger} \hat{a}_{u}\right\rangle \\
& =\left(N^{2}+N\right) / 2,
\end{aligned}
$$

where $\left\langle\hat{a}_{u}^{\dagger} \hat{a}_{u}\right\rangle=N$ and $\left\langle\hat{a}_{u}^{\dagger} \hat{a}_{u} \hat{a}_{u}^{\dagger} \hat{a}_{u}\right\rangle=\left(3 N^{2}+N\right) / 2$. From Eq.(1), we find the phase fluctuation $\Delta \phi \sim$ $\sqrt{2} / \sqrt{N^{2}+N}$. Thus if we use this state to probe a small phase shift, the measurement precision may approach $\sqrt{2} / N$ when $N$ is much larger than 1 . This is close to the Heisenberg limit of $1 / N$.

Let us now see how we can use the above state for phase measurement. When the phase shift operator $\hat{U}=$ $\exp \left(i \phi \hat{a}_{u}^{\dagger} \hat{a}_{u}\right)$ acts on this state, the state becomes:

$$
\begin{gathered}
\left|\Psi_{2 N}(\phi)\right\rangle=\sum_{k=0}^{N} \frac{(-1)^{N-k}}{2^{N}}\left[\left(\begin{array}{c}
2 k \\
k
\end{array}\right)\left(\begin{array}{c}
2 N-2 k \\
N-k
\end{array}\right)\right]^{1 / 2} \\
\times e^{i 2 k \phi}|2 k\rangle_{u}|2 N-2 k\rangle_{d} .
\end{gathered}
$$

Refs. [8, 11] provided two methods to extract the phase information: one is based on the variance of the photon number difference [8] while the other on a parity measurement [11]. However, none of them are easily implemented experimentally. Here we present a different method for phase measurement, based on the general guideline outlined in Ref. [6]. The idea is to compare the phase-shifted state in Eq.(5) with the original unshifted one in Eq.(3). This can be done by making the projection of the state $\left|\Psi_{2 N}(\phi)\right\rangle$ onto the state $\left|\Psi_{2 N}\right\rangle:\left\langle\Psi_{2 N} \mid \Psi_{2 N}(\phi)\right\rangle$, which is simply the measurement of the projection operator $\hat{\mathbb{P}} \equiv\left|\Psi_{2 N}\right\rangle\left\langle\Psi_{2 N}\right|$ on the state $\left|\Psi_{2 N}(\phi)\right\rangle$ :

$$
\begin{aligned}
P(\phi) & =\left\langle\Psi_{2 N}(\phi)|\hat{\mathbb{P}}| \Psi_{2 N}(\phi)\right\rangle=\left|\left\langle\Psi_{2 N} \mid \Psi_{2 N}(\phi)\right\rangle\right|^{2} \\
& =\left\{\sum_{k=0}^{N} \frac{\cos [\phi(2 k-N)]}{2^{N}}\left(\begin{array}{c}
2 k \\
k
\end{array}\right)\left(\begin{array}{c}
2 N-2 k \\
N-k
\end{array}\right)\right\}^{2} .
\end{aligned}
$$

The projection operator $\hat{\mathbb{P}} \equiv\left|\Psi_{2 N}\right\rangle\left\langle\Psi_{2 N}\right|$ can be constructed by the method described in Ref.[16]. From the identity equation

$$
\begin{aligned}
& \sum_{k=0}^{N}(-1)^{N-k} x^{2 k} y^{2(N-k)}\left(\begin{array}{l}
N \\
k
\end{array}\right)\left(\frac{1}{2}\right)^{2 N} \\
& =\left(x^{2}-y^{2}\right)^{N} / 2^{N}=[(x-y) / \sqrt{2}]^{N}[(x+y) \sqrt{2}]^{N},
\end{aligned}
$$

we find that the projection $\hat{\mathbb{P}}$ is achieved by measuring the probability of detecting $N$ photons at each outport of a 50:50 beam splitter $\mathrm{BS}_{2}$ (Fig.1b). The $2 \mathrm{~N}$-photon coincidence rate is proportional to $P(\phi)$ in Eq.(6)

The phase uncertainty from the error propagation theory is given by [17]

$$
\Delta \phi=\Delta P(\phi) /\left|\frac{\partial P(\phi)}{\partial \phi}\right|,
$$

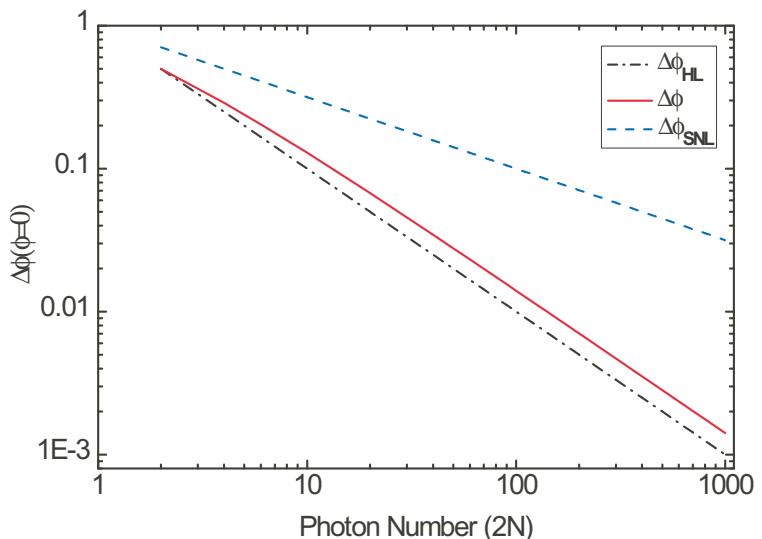

FIG. 2: Phase uncertainty versus photon number $N$. The solid line shows the phase uncertainty at $\phi=0$ using the twinphoton state input and the projection measurement. The dash-dotted line and dashed line show the Heisenberg limit $\Delta \phi_{H L}$ and the standard quantum limit $\Delta \phi_{S Q L}$.

where $\Delta P(\phi) \equiv\left\langle\hat{\mathbb{P}}^{2}\right\rangle-\langle\hat{\mathbb{P}}\rangle^{2}=P(\phi)[1-P(\phi)]$. For the case of $N=1$, the state $\left|\Psi_{2 N}\right\rangle$ is exactly the two-photon MES state and $P(\phi)=\cos ^{2} \phi$ so that the phase uncertainty defined in Eq.(8) is $\Delta \phi=1 / 2$. This is exactly the Heisenberg limit for two photons. With $N=2$, $P(\phi)=\left(\frac{3}{4} \cos 2 \phi+\frac{1}{4}\right)^{2}$, and $\Delta \phi=1 / \sqrt{12}=0.289$ in the limit $\phi \rightarrow 0$. This is slightly larger than the Heisenberg limit of $\Delta \phi_{H L}=1 / 4=0.25$, but is much less than the standard quantum limit of $\Delta \phi_{S Q L}=1 / \sqrt{4}=0.5$ for four photons. A log-log plot of the phase uncertainty derived from Eqs. (6. 8) with $\phi=0$ versus photon number $N$ is shown in Fig.2 as the solid line together with the Heisenberg limit (dash-dotted line) and the standard quantum limit (dashed line), respectively. With a large photon number, the slope of the $\Delta \phi$ is approaching -1 , just as the slope of $\Delta \phi_{H L}$. However, the plot of $\Delta \phi_{S Q L}$ shows a slope of $-1 / 2$. It is clear that $\Delta \phi$ is much better than $\Delta \phi_{S Q L}$. From the numerical calculation, we have $\Delta \phi \sim 1.4 \Delta \phi_{H L}$ for a large photon number.

Experiment - Compared to the MES, the two-mode twin Fock state and the state $\left|\Psi_{2 N}\right\rangle$ in Eq.(3) after the beam splitter can be prepared more easily. For experimental implementation, two orthogonal polarization modes are used instead of the spatial modes in Fig.1. The polarization twin Fock state can be generated through the process of type-II parametric down conversion (PDC). As shown in Fig.3, a BBO crystal cut for type-II beam-like parametric down-conversion [18] is pumped by $150 \mathrm{fs}, 200 \mathrm{mw}$ ultraviolet $(390 \mathrm{~nm})$ pulses to produce the photon pairs. The down converted photons $(780 \mathrm{~nm})$ are coupled into the polarization maintaining single-mode fiber. The two polarization modes are combined by a polarization beam splitter $\left(\mathrm{PBS}_{1}\right)$ and transmitted through a $3 \mathrm{~nm}$ interference filter (IF) and a half wave plate $\left(\mathrm{HWP}_{1}\right)$.

It is easy to implement for photon states with small 


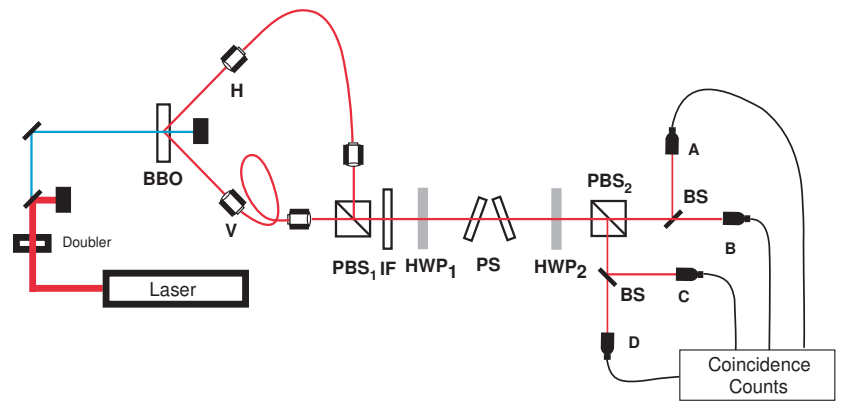

FIG. 3: (color online) Experimental setup. PBS: polarization beam splitter; HWP: half wave plate; IF: interference filter; PS: phase shifter; BS: beam splitter.

numbers such as $N=1,2$, which can be realized with two and four detectors, respectively (Fig.3). The phase shift operator $\hat{U}=\exp \left(i \phi \hat{a}_{H}^{\dagger} \hat{a}_{H}\right)$ on horizontal polarization is realized by changing the incident angles of the two identical quartz crystals (PS), as illustrated in Fig.3. To avoid the shift of the beam, the two quartz crystals are turned simultaneously in opposite directions. The projection measurement of $\left|\Psi_{2 N}\right\rangle$ is achieved by $2 \mathrm{~N}$-fold coincidence measurement after the half wave plate $\mathrm{HWP}_{2}$ (set at $22.5^{\circ}$ ) and $\mathrm{PBS}_{2}$. The detection of $N$ photons in each output port of $\mathrm{PBS}_{2}$ indicates a successful projection to $\left|\Psi_{2 N}\right\rangle$. For two-photon case, detectors A and $\mathrm{C}$ are used while all four detectors are involved for fourphoton case.

Fig.4 shows the results of measurement with twophoton and four-photon states. The data are normalized to the maximum count. The result for two-photon state is illustrated in Fig.4(a). The data are fitted to the function of $P_{2}(\phi)=(1+V \cos 2 \phi) /(1+V)$, with $V=(95.3 \pm 0.1) \%$ as the visibility defined by $V \equiv$ $\left(C_{\max }-C_{\min }\right) /\left(C_{\max }+C_{\min }\right)$. So the minimal phase uncertainty defined in Eq.(8) is $\Delta \phi=0.506 \pm 0.001$ when $\phi=0$, whereas for an average photon number of 2 , the standard quantum limit is $\Delta \phi_{S Q L}=0.707$.

Fig.4(b) shows the result of four-photon case. Since the four photons are from two pairs of down-converted photons, we need to consider the distinguishability between the two pairs [19]. Then the result is [20]

$$
P_{4}(\phi)=\frac{(1+2 \mathcal{E} / \mathcal{A})(3 \cos 4 \phi+4 \cos 2 \phi)+9+2 \mathcal{E} / \mathcal{A}}{16+16 \mathcal{E} / \mathcal{A}},
$$

where $\mathcal{E} / \mathcal{A}(\leq 1)$ describes the degree of temporal distinguishability between two pairs of photons. The closer to one the value of $\mathcal{E} / \mathcal{A}$ is, the more indistinguishable the pairs are. In Fig.4(b), the solid curve is a least square fit to the above function with $\mathcal{E} / \mathcal{A}=0.93 \pm 0.03$. This value of $\mathcal{E} / \mathcal{A}$ is consistent with that in previous study [18]. From $P_{4}(\phi)$, we may derive the phase uncertainty from Eq.(8) and plot it against the phase shift $\phi$, as shown in Fig.5. Compared with the standard quantum limit (dashed), the phase uncertainty (a)
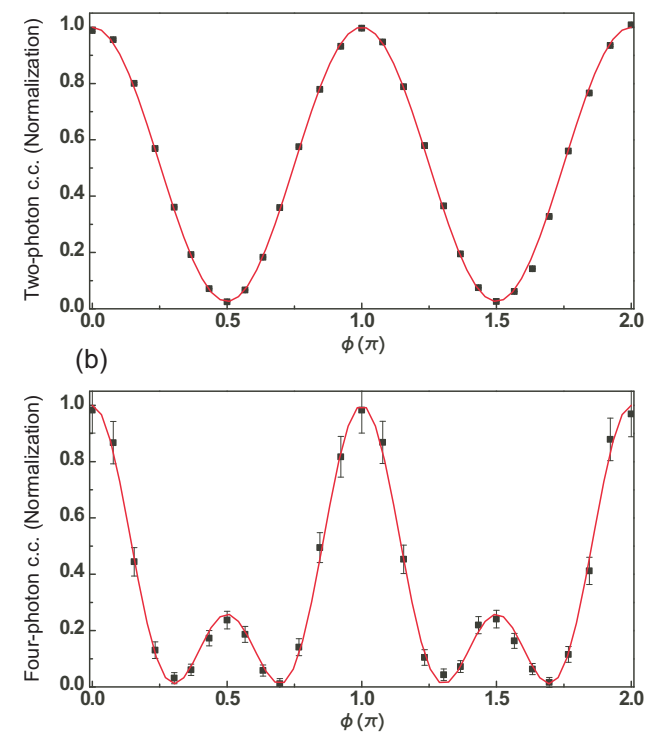

FIG. 4: (color online) Experiment data: (a) Two-photon coincidence counts between detectors $\mathrm{A}$ and $\mathrm{C}$ versus the singlephoton phase difference $\phi$. The continuous curve is a least square fit to the function $P_{2}(\phi)$. (b) Four-photon coincidence counts versus $\phi$. They are fitted to the function of $P_{4}(\phi)$. The data are normalized to the maximum counts of $8837 / \mathrm{s}$ and $375 / 100 s$ for (a) and (b), respectively.

from the four-photon state is better in the regions of $\phi \in(k \pi-0.885, k \pi+0.885)$. The minimal phase uncertainty is $\Delta \phi_{\min }=0.291 \pm 0.001$ at $\phi=k \pi$.

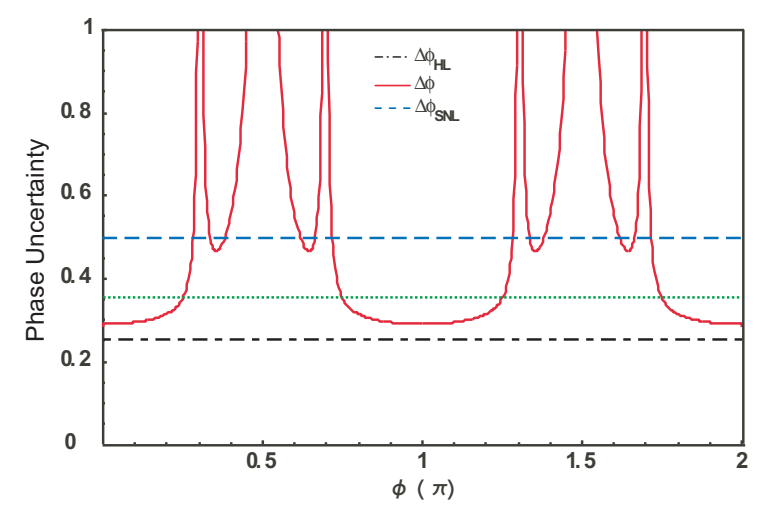

FIG. 5: (color online)Phase uncertainty versus phase shift. The solid line shows the phase uncertainty using the fourphoton state. Correspondingly, the dash-dotted line and dashed line show the cases of Heisenberg limit $\Delta \phi_{H L}$ and standard quantum limit $\Delta \phi_{S Q L}$.

The improvement of precision comes from the multiphoton interference effect due to multi-photon entanglement. The optimal state is the MES. Other states with less entanglement, such as the two-mode state in Eq.(3), may show a phase measurement precision worse than the 
MES, but they may still give a precision close to the Heisenberg limit. However, decoherence in the multiphoton state will reduce the entanglement effect and influence the phase measurement precision. For example, if the four photons state from the parametric down conversion are separated into two distinguishable pairs, i.e. $\mathcal{E} / \mathcal{A}=0$ [19], the theoretical phase uncertainty is then 0.354 (shown as the dotted line in Fig.5). The value of 0.354 is still better than the standard quantum limit $\Delta \phi_{S Q L}=0.5$. This is because there is still some partial entanglement in this four-photon state (the downconverted two photons are correlated and entangled).

In experiment, losses of photons and imperfection in detector efficiency will decrease the success probability of projection measurement. However, it will not affect the uncertainty of phase measurement because the measurement fidelity remains unchanged. The loss in projection measurement can be modelled as: $\mathbb{M}=$ $\eta^{2}\left|\Psi_{2 N}\right\rangle\left\langle\Psi_{2 N}\right|$, where the coefficient $\eta$ depends on photon losses and detector efficiency. In the calculation of $\Delta \phi$, we have $\Delta^{2} M(\phi)=\left\langle\Psi_{2 N}(\phi)\left|\mathbb{M}^{2}\right| \Psi_{2 N}(\phi)\right\rangle-$ $\left(\left\langle\Psi_{2 N}(\phi)|\mathbb{M}| \Psi_{2 N}(\phi)\right\rangle\right)^{2}=\eta^{4}\left[P(\phi)-P^{2}(\phi)\right]$ and $\left|\frac{\partial M(\phi)}{\partial \phi}\right|=\eta^{2}\left|\frac{\partial P(\phi)}{\partial \phi}\right|$. From Eq.(8), we find that the uncertainty of the phase measurement is unchanged. The reason for this is that projection measurement is a postselection of the states with a fixed photon number for the input state. Lost photons are excluded. However, the higher photon number states that may arrive from higher order parametric down-conversion would contribute to the background and should be subtracted. Otherwise it will decrease the fidelity of projection measurement.
Therefore, loss only decreases the success probability and thus increases the number of trials required, that is we will need $N / \eta$ photons instead of $N$ photons. But as long as $\eta$ is fixed, the phase uncertainty will be $1.4 / \eta N \sim 1 / N$, similar to the Heisenberg limit. Multi-photon coincidence measurement usually has very low efficiency. It is anticipated that as technology develops [21], new efficient multi-photon detector will replace the low efficiency multi-photon coincidence measurement for the projection measurement. Since our experiment is a demonstration of principle, we set $\eta$ to 1 in our analysis.

In conclusion, we discussed a new state projection measurement for extracting the phase information with a twin Fock state, with a for large photon number. The concept, i.e., a state preparation for phase measurement and a projection for comparison, can be generalized. Experimentally, we demonstrate the feasibility of the scheme with a phase measurement precision of $0.506 \pm 0.001$ for two-photon state and $0.291 \pm 0.001$ for four-photon state, respectively. Unlike many other schemes realized so far [12, 13, 15, 16, 22], our scheme can be applied to large photon numbers.

After submitting our manuscript, we became aware of the work by Nagata et al. on a similar topic [22].

\section{Acknowledgments}

This work was funded National Fundamental Research Program, the Innovation funds from Chinese Academy of Sciences, National Natural Science Foundation of China (Grant No.60121503). ZYO is also supported by the US National Science Foundation under Grant No. 0427647.
* Electronic address: fwsun@mail.ustc.edu.cn

$\dagger$ Electronic address: zou@iupui.edu

[1] P. A. M. Dirac, Proc. R. Soc. London Ser. A 114, 243 (1927); W. Heitler, The Quantum Theory of Radiation, 3rd ed. (Oxford University Press, London, 1954), p. 65.

[2] C. M. Caves, Phy. Rev. D 23, 1693 (1981).

[3] R. S. Bondurant and J. H. Shapiro, Phy. Rev. D 30, 2548 (1984).

[4] M. Xiao, L. A. Wu, and H. J. Kimble, Phys. Rev. Lett. 59, 278 (1987).

[5] Z. Y. Ou, Phys. Rev. Lett. 77, 2352 (1996).

[6] Z. Y. Ou, Phys. Rev. A. 55, 2598 (1997).

[7] W. Heisenberg, Zeitschr. f. Physik 43, 172 (1927).

[8] M. J. Holland and K. Burnett, Phys. Rev. Lett. 71, 1355 (1993).

[9] J. Jacobson, G. Björk, I. Chuang, and Y. Yamamoto, Phys. Rev. Lett. 74, 4835 (1995).

[10] J. J. Bollinger, W. M. Itano, D. J. Wineland, and D. J. Heinzen, Phys. Rev. A. 54, R4649 (1996).

[11] R. A. Campos, C. C. Gerry, and A. Benmoussa, Phys. Rev. A. 68, 023810 (2003).

[12] P. Walther, J. -W, Pan, M. Aspelmeyer, R. Ursin, S. Gasparoni, and A. Zeilinger, Nature(London) 429, 158 (2004).

[13] M. W. Mitchell, J. S. Lundeen, and A. M. Steinberg,
Nature(London) 429, 161 (2004).

[14] A. N. Boto, P. Kok, D. S. Abrams, S. L. Braunstein, C. P. Williams, and J. P. Dowling, Phys. Rev. Lett. 85, 2733 (2000).

[15] K. J. Resch, K. L. Pregnell, R. Prevedel, A. Gilchrist, G. J. Pryde, J. L. OBrien, and A. G. White, Phys. Rev. Lett. 98, 223601 (2007).

[16] F. W. Sun, Z. Y. Ou, and G. C. Guo, Phys. Rev. A 73, 023808 (2006).

[17] V. Giovannetti, S. Lloyd, and L. Maccone, Science 306, 1330 (2004).

[18] G. Y. Xiang, Y. F. Huang, F. W. Sun, P. Zhang, Z. Y. Ou, and G. C. Guo, Phys. Rev. Lett. 97, 023604 (2006).

[19] Z. Y. Ou, J.-K. Rhee, and L. J. Wang, Phys. Rev. A 60, 593 (1999).

[20] Because the two-photon interference has high visibility, we do not consider photon distinguishability within one pair of photons when we deduce $P_{4}(\phi)$.

[21] J. Kim, S. Takeuchi, and Y. Yamamoto, Appl. Phys. Lett. 74, 902 (1999); E. Waks, E. Diamanti, and Y. Yamamoto, New J. Phys. 8, 4 (2006).

[22] T. Nagata, R. Okamoto, J. L. O'Brien, K. Sasaki, and S. Takeuchi, Science, 316, 726 (2007). 